“드 2013 IEEE. Personal use of this material is permitted. Permission from IEEE must be obtained for all other uses, in any current or future media, including reprinting/republishing this material for advertising or promotional purposes, creating new collective works, for resale or redistribution to servers or lists, or reuse of any copyrighted component of this work in other works." 


\title{
CaptureMyEmotion: a Mobile App to Improve Emotion Learning for Autistic Children Using Sensors
}

\author{
Peter Leijdekkers, Valerie Gay and Frederick Wong \\ Faculty of Engineering and Information Technology, University of Technology, Sydney, \\ PO Box 123, Broadway 2007, NSW, Australia. \\ Peter.Leijdekkers@uts.edu.au
}

\begin{abstract}
Autism Spectrum Disorder (ASD) is estimated to affect one in eighty-eight children and many mobile apps are available from Google Play or Apple store to help these children and their carer. Our research into apps for autistic children identified that none of the apps use the full potential offered by mobile technology and sensors to overcome one of autistic children's main difficulty: the identification and expression of emotions. This paper describes a mobile app called CaptureMyEmotion that enables autistic children to take photos, videos or sounds, and at the same time senses their arousal level using a wireless sensor. It also allows the child to comment on their emotion at the time of capture. The app has the potential to help autistic children improve their emotions learning based on their own pictures, videos or sounds. It gives the carer a means to discuss the identification and expression of emotions.
\end{abstract}

\section{Introduction}

The Autism Spectrum Disorder (ASD) describes a range of conditions typically characterized by social deficits, communication difficulties, stereotyped or repetitive behaviours and interests, and in some cases, cognitive delays [1]. The prevalence of ASD is estimated to be up to 1 in 88 children [2]. Understanding and managing emotions is very challenging for the majority of autistic children and it is of major importance for their integration in classrooms and other social groups.

Autistic children, like other children of their generation, embrace mobile technology and enjoy using smart phones and tablets to learn and play. Most autistic children are visual learners and mobile technology appeals to them [3]. In our research at the University of Technology, Sydney we investigate how mobile technology and sensors can help autistic children and their carer understand and manage emotions.

It is increasingly recognised that autistic children respond well to personalised technology [4-6]. Autistic associations such as FaHCSIA [7] in Australia or Autism Speaks [8] in the USA have identified the potential of mobile technology on the child's progress and are subsidising or giving tablets away to families with autistic children.

Mobile technology has several advantages for the autistic child and carer:

- Access to tools to play and communicate ubiquitously.

- Easy access to games, music, education tools on the go.

- The carer can easily identify what the child likes to play with and track progress.

- Multimodal input/output with an intuitive interface.

- Easy use of camera and video.

- Easy integration of external sensors that wirelessly transmit data to the device providing additional feedback.

- An action (e.g. taking a picture) can be positioned in context using GPS, time and other data, which can be used at a later stage.

- Using one of the many apps available, the carer can help manage social/emotional skills and behaviour management.

- Mobile devices include features to provide better accessibility for autistic children (e.g. extra large font size, disabling buttons) [9].

Hundreds of Apps exist to help autistic children and their carers or teachers. At the time of writing (January 2013) a search on 'autistic app' returned $1000+$ results in Google Play [10]. However, none of these apps use sensor input to measure the emotion of the user. Many autistics have difficulties in expressing or communicating their emotions/feelings in a similar way to how non-autistic people would normally do [11]. Many apps for people with autism or other 
special needs have been developed for the iPhone and Android platform. A good overview can be found in [12] and [13].

This paper presents CaptureMyEmotion, an App for smartphones and tablets, which uses Affectiva's Q sensor to help autistic children gauge the intensity of their emotions. CaptureMyEmotion enables children to take photos, videos or sounds and, at the same time, sense and self report their emotion. The collected data gives the carer insight into the child's emotions and offers a means to discuss the identification and expression of emotions. What sets CaptureMyEmotion apart is that we combine personally recorded photos, videos or sounds together with contextual information (date, time, location) and emotion data measured by a sensor. This creates a very personalised tool for a child to learn and discuss their feelings. Sensor data is used when the child takes a picture, as well as, when the child discusses the picture with a carer at a later stage. The use of an emotion sensor adds a new dimension to emotion learning by providing additional feedback based on physiological data.

\section{Emotions and sensors}

According to Schlosberg [14], emotion can be described by two dimensions i.e. arousal (high versus low) and valence (positive versus negative). Based on these two dimensions, 6 basic emotions (disgust, sadness, happiness, fear, anger, surprise) can be identified which exist for all human beings. For example, anger can be classified as high arousal and negative valence.

There is no perfect measure of arousal or valence. However, good approximations can be obtained by measuring arousal from the sympathetic nervous system (SNS) and valence from facial expressions [15].

Nervousness, fear, anger, protectiveness, and lust are enhanced by the sympathetic nervous system and can be measured through physiological changes in the body. Typically, physiological signals such as electrocardiogram (ECG), electromyography (EMG), blood volume pulse, skin temperature, skin conductance or respiration are used to measure arousal [16].

Using physiological signals to recognize emotion has several advantages compared to other methods such as speech and face recognition. Sensors capturing physiological signals are nowadays compact and often allow wireless transmission of the data to other devices such as smartphones or tablets. The sensors are comfortable to wear and are less obtrusive than, for example, being observed by a camera to be able to do facial expression recognition.

Skin temperature measures the temperature on the surface of the skin. When muscles are tense under strain, the blood vessels will be contracted and therefore the temperature will decrease. It is a relatively slow indicator of changes in emotional state.

Skin conductance or electrodermal activity (EDA) is an important signal to measure arousal. Electrodermal activity refers to changes in the skin's ability to conduct electricity. If a person experiences emotional arousal, increased cognitive workload or physical exertion, the brain sends signals to the skin to increase the level of sweating. Skin conductance usually increases when a person is more aroused (engaged, stressed, or excited) and it tends to stay low or drop when a person is less aroused (disengaged, bored, or calm). To detect changes in emotional state using skin conductance, it is important to obtain a baseline indicating the truly lowest tonic skin conductance level. The best way to get a baseline is to measure long-term, and look for the lowest smooth period of skin conductance where there is no physical movement, and where the temperature is clearly body temperature [15].

CaptureMyEmotion uses a wrist worn sensor called the $Q$ sensor from Affectiva (www.affectiva.com). The $Q$ Sensor measures changes in skin temperature, motion, and skin conductivity, and is able to give an indication about the wearer's emotional state. The sensor is easy to wear and is able to transmit the data in real time to a mobile phone. The $Q$ sensor can be worn for a long period of time to obtain a reliable baseline.

A challenging issue is to measure valence. Traditionally, people would rate their feelings along the valence-arousal dimensions. However, self-rating can be highly inaccurate for many autistics so a more objective way needs to be found. Detecting the facial expression automatically can be the solution and one idea is to take a picture of the child when he rates a picture just taken. However, many autistics do not show a particular facial expression so this can be unreliable.

\section{CaptureMyEmotion}

The app is used to assign emotions to pictures, videos or sounds taken by the child. Our target group is children from 7 years and older who are able to use a smartphone to take pictures. CaptureMyEmotion is different from many existing apps since it allows the user to take their own pictures (When we refer to pictures this also includes videos or sounds) and not based on a fixed set of pictures. This will increase the 
chance that a picture generates a genuine emotion. Additionally, the app can be useful to obtain visual evidence that correlates to a particular emotion as measured by the sensor or self-reporting. For example, the user can be asked to take pictures of objects that make him happy. The app will provide a history of pictures categorized by a particular emotion and this gives the carer an insight into the child's emotions.

Our extensive experience with wireless sensors in health related mobile apps such as myFitnessCompanion ${ }^{(2)}$ (myFitnessCompanion.com) has shown that most users want to have the option to use a sensor or not. The reasons can be diverse and range from cost (too expensive!) to practicality (sensor not at hand). Therefore, the sensor is optional and the app asks the child to manually select the emotion felt just after the picture has been taken. This is useful information, which can lead to valuable discussions with the carer even if the sensor is not used.

If the user wears the $Q$ sensor, the sensor collects in real time accelerometer, skin temperature and skin conductive data associated with the picture taken. Based on the physiological data, the app calculates the arousal level and stores it together with the picture and contextual information (date, time and GPS coordinates). The $\mathrm{Q}$ sensor is also used during the discussion with the carer, and it keeps track of the child's arousal level while discussing the picture.

CaptureMyEmotion is developed for both Android smartphones and tablets. The child would typically use the smartphone to take pictures and the carer would use the tablet for evaluation together with the child. Tablets are more suitable for this due to the amount of data displayed. CaptureMyEmotion uses Dropbox to share the data between several devices. When the child takes a picture, it is automatically uploaded to a Dropbox account via $3 \mathrm{G}$ or WiFi. If the carer deals with several children (e.g. in a classroom situation), the carer can synchronize the data for the various children using Dropbox on the tablet.

The child can select camera, video or sound to capture the object that is in the viewer. The app automatically connects to the $\mathrm{Q}$ sensor (if present) and a gauge icon displays, in real-time, the arousal level experienced by the child. The arousal range is stored after a picture has been taken.

After a picture is taken, a screen is shown where the child can select a basic emotion using personalised pictures to represent the various emotions. When an emotion is selected, a sound mimicking the selected emotion is played (e.g. a laughing sound for 'happy'). Sound can be disabled in the settings. When the done button is tapped, the selected emotion is stored together with the Q sensor data and automatically uploaded to the Dropbox account if activated.
CaptureMyEmotion allows the child or carer to set the language, as well as, the images for the emotions. Some children prefer icons (smiley type of images) to real pictures [17].

Pictures, contextual and sensor data are automatically downloaded to the tablet when Dropbox sharing is activated. By default, the pictures are organised in chronological order for each day. This might help reinforce the learning of narrative, which has been observed as difficult for autistic children [18]. Pictures, videos and sounds can also be organised based on date, carer-rating and self reported emotion.

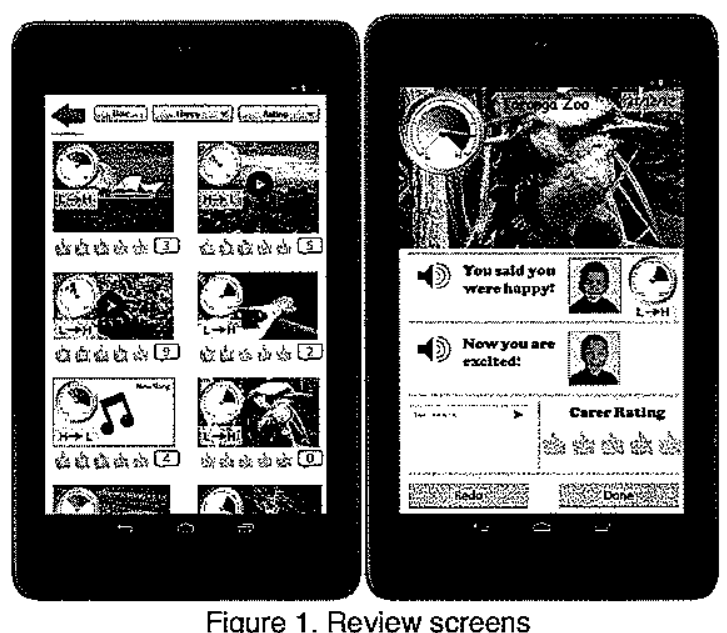

Figure 1 (right) shows pictures filtered on 'happy'. Under each picture, it shows the carer rating (thumbs), the number of views and the arousal level (gauge) around the time the picture was taken.

When a picture is selected for review, the child can assign an emotion to the picture again and the next screen shows a summary (Figure 1 right). The summary shows the current emotion coupled with a real-time feed of the arousal level of the user. It also shows the emotion selected by the child at the time the picture was taken together with the arousal range detected then.

When reviewing the pictures, the child has the opportunity to express their feelings toward a certain picture in the company of a carer. The use of the picture, selected emotion and arousal gauge helps the child to overcome the difficulty of communicating with another person. This works in a similar way to the picture card method for children who might not be able to fully verbalise their emotions [19]. Therefore, the review gives ample opportunity to discuss the difference in the emotion felt during review compared to the emotion felt immediately after the picture was taken. This discussion could allow the child to delve 
into the reasons behind why he felt a certain way at a certain time.

If the child wears the sensor, the real-time arousal level is shown as detected by the $Q$ sensor. This gives the carer insight into what emotion the picture evokes, or can highlight that the child is getting tired of doing the review or being distracted by other external factors. This can be used in the discussion with the child, or as an indicator that the carer should switch to another activity.

The carer can comment and rate the picture as seen fit. The carer rating (number of thumbs-up) allows the carer to express the review result of a picture with the child (e.g.: 0-no review, 1-very poor, 5-very good).

Data accumulated from the carer and user can be presented in progress reports. For example, a graph detailing the total number of thumbs accumulated against time. The child/carer is also able to see how many times a certain rating (i.e. number of thumbs) has been awarded over a certain period of time. Furthermore, the user is able to view the number of thumbs received filtered on a particular emotion, location or comments.

\section{Conclusion}

This paper presented an overview of CaptureMyEmotion, an app for smartphones and tablets. It aims to help autistic children understand their emotions with the support of a mobile device and sensors. Since every child is different, the frequency of use of the app, and the number of feedback sessions highly depends on the child's interest and capabilities, as well as, on the carer's objectives. CaptureMyEmotion is a tool and (by choice) it allows the carer choose what they want to do with the collected information. For example, discuss what the child did during the day, understand emotions and identify what makes the child react.

Understanding and managing emotions is important for autistic children since it has an impact on how well they integrate into society.

We plan to trial CaptureMyEmotion on autistic and no autistic children to compare the autistic children's reaction to the app against a baseline from non-autistic children. Based on feedback received we will improve the app and then make it available on Google Play.

\section{References}

All websites have been viewed on the $1^{\text {st }}$ of May 2013 .

[1] “Diagnostic and Statistical Manual of Mental Disorders", American Psychiatric Association 4th edition, 2000.
[2] J. Baio et al, "Prevalence of Autism Spectrum Disorders - Autism and Developmental Disabilities Monitoring Network, 2008", MMWR 61(SS03), March 2012; 1-19.

[3] Z.S. de Urturi, A.M. Zorrilla, and B.G. Zapirain, "Serious Game based on first aid education for individuals with Autism Spectrum Disorder using android mobile devices", CGAMES USA, July 2011, 223-227.

[4] W. Farr, "Personalised technology for autism spectrum conditions is the future", Journal of Assistive Technologies, volume 4 , issue $1,2010,58-60$.

[5] R.W. Picard and M.S. Goodwin, "Innovative technology: the future of personalized autism research and treatment". Autism Advocate 1 (1), 2008, 32-39.

[6] H. van Rijn and P.J. Stappers, "Codesigning Linkx; a case of gaining insight in a difficult-to-reach user group". IASDR07, November 2007, 1-14.

[7] FaHCSIA, "early intervention funding", www.fahcsia.gov.au .

[8] Autism speaks, "iPads Sent to 651 Financially Disadvantaged Families", December 2012. www.antismspeaks.org.

[9] Apple website, "IOS 6-Accessibility", www.apple.com .

[10]Google Play website, www.play.google.com/store/apps .

[11]Autism speaks, "11 Myths About Autism", http://blog.autismspeaks.org .

[12]iAutism, www,iautism,info/en/category/application-lists.

[13]Autism speaks, www.autismspeaks.org/autism-apps.

[14]Schlosberg, "Three Dimensions of Emotion", The Psychological Review, Volume 61, No 2, March 1954, 8188.

[15]R. W. Picard, "Future affective technology for autism and emotion communication", Phil. Trans. R. Soc. B, 2009, 3575-3584

[16]S. Jerritta, M.Murugappan, R. Nagarajan, and Wan, K, "Physiological signals based human emotion Recognition: a review," CSPA 2011, March 2011, 410-415.

[17]Flourish $\mathrm{n}$ thrive counseling, "therapy tools and counseling resources", flourishnthrive.wordpress.com.

[18]O. Sacks, "An Anthropologist on Mars". Picador, London, 1996.

[19]W. Barendregt, M.M. Bekker, E. Baauw, "Development and evaluation of the problem identification picture cards method", Cognition, Technology and Work, 2008, 95-105 